\title{
Rezension „Augmented Reality im E-Commerce“
}

\author{
Stefan Stieglitz
}

Angenommen: 23. Dezember 2021 / Online publiziert: 17. Januar 2022

(C) Der/die Autor(en) 2022

Frank Huber, Cecile Kornmann und Sandra Wolnitz Augmented Reality im E-Commerce - Die Vorteile der Technologie für den Online-Handel

ISBN 978-3-96146-733-4, Diplomica Verlag, Hamburg, 2019, Reihe Marketing Band 1, 142 S., 42,50€

Die Digitalisierung revolutioniert nicht nur das Einkaufserlebnis durch Online-Stores, in denen Unternehmen ihre Produkte präsentieren können, sie bringt auch neue Technologien hervor, die von Unternehmen eingesetzt werden können, um möglichen Kundinnen und Kunden ein verbessertes Einkaufen zu ermöglichen. Unter anderen können sich Unternehmen Augmented Reality Technologien zu Nutze machen, um ihre Produktpräsentation zu unterstützen. Sowohl virtuelle als auch Augmented Reality-Technologien werden in vielen verschiedenen Bereichen immer beliebter und entwickeln sich stetig weiter. Die Autoren Frank Huber, Cecile Kornmann und Sandra Wolnitz haben somit ein Forschungsthema am Puls der Zeit gefunden, indem sie den Einsatz von Augmented Reality in Online-Stores untersuchen, da beide Technologien immer mehr an Relevanz gewinnen. Zudem kann eine der größten Herausforderungen, die Online-Stores begegnen, durch Augmented Reality adressiert werden. Im Gegensatz zu herkömmlichen Stores können die Produkte nicht anprobiert, angefasst oder ausprobiert werden. Da Augmented Reality virtuelle Objekte mit der realen Umwelt verbindet, kann damit diesem Problem entgegengewirkt werden. So können Kleidungsstücke auf ein virtuelles Abbild der eigenen Person projiziert oder Möbelstücke in der Wohnung virtuell platziert werden. Das übergeordnete Ziel des Einsatzes von Augmented Reality ist es also, die Zufriedenheit des Kauferlebnis der Kundinnen und Kunden zu verbessern.

Stefan Stieglitz $(\square)$

Universität Duisburg-Essen, Duisburg, Deutschland

E-Mail: stefan.stieglitz@uni-due.de 
Die Autorinnen und Autoren des Buches haben sich ein relevantes und aktuelles Forschungsthema ausgesucht und empirisch untersucht. Genauer gesagt wurde im Rahmen des wissenschaftlich fundierten Buches untersucht, wie Augmented Reality (unterteilt in eine erweiterte Form der Darstellung eines Kaufprodukts oder in eine Möglichkeit der Interkation mit dem Kaufprodukt) Kauferlebnis, wahrgenommenes Risiko und Entscheidungssicherheit beeinflusst. Gleichzeitig wurden Kaufmotivation und Shopping Enjoyment als weitere Einflussfaktoren berücksichtigt. Um Augmented Reality im E-Commerce besser zu verstehen, wurde ein Online-Fragebogen entwickelt, der die genannten Konstrukte abfragt und für den Online-Shopping Szenarien entwickelt wurden, in denen Proband: innen sich vorstellen müssen, Augmented Reality für den Kauf von Kleidung einzusetzen. Getestet wurden die Annahmen mit einem Datensatz bestehend aus 308 Fällen durch eine Varianzanalyse oder Regressionsanalyse, abhängig vom Forschungsgegenstand und Formulierung der Hypothesen. Es zeigte sich, dass der Einsatz von Augmented Reality unabhängig von den Moderatorvariablen einen signifikanten Einfluss auf das wahrgenommene Risiko hat. Das Kauferlebnis und Entscheidungssicherheit wurden durch den Einsatz von Augmented Reality nur signifikant beeinflusst, wenn eine Differenzierung der Konsumentengruppen erfolgte.

Das Buch startet damit, dass die Relevanz des Einsatzes von Augmented Reality in Online-Stores dargestellt und erläutert wird. So wird deutlich, dass Online-Shopping immer beliebter bei den Konsumentinnen und Konsumenten wird, es aber auch häufig zu Kaufabbrüchen kommt. Gleichzeitig wird die wachsende Wichtigkeit der Augmented Reality Technologie dargelegt sowie die Vorteile, die Augmented Reality für den E-Commerce mit sich bringen kann. In diesem Kontext wird deutlich, dass es noch wenig empirische Erkenntnisse über den Einsatz von Augmented Reality in Online-Stores gibt, was erneut die Relevanz des Buches und dessen Studie unterstreicht. Es werden zudem relevante Theorien und Modelle für die psychologischen Mechanismen beim Online-Shopping vorgestellt, wie zum Beispiel die Customer Experience, das wahrgenommene Risiko und die Decision Confidence. Somit bekommen die Leser:innen des Buches bereits mit dem ersten Kapitel ein breites Verständnis für die Forschungslücke, die im Rahmen des Buches untersucht wird, aber auch eine erste Einleitung in die untermauernden Konstrukte, die im Zusammenhang mit der Forschungslücke wichtig sind. Die Autor: innen kreieren somit einen hervorragenden Start in das Buch, da sowohl die Forschungslücke, die Relevanz der Forschungslücke und nötige Hintergrundinformationen gut dargelegt werden.

Im zweiten Kapitel werden die genannten Theorien und Modelle im Detail vorgestellt. Es beginnt damit, dass die Leser:innen weitere Informationen zu den Eigenschaften von Augmented Reality bekommen, sowie zum Nutzen von Augmented Reality in Online-Stores. Es wird dazu übergegangen, wie Online-Stores ohne Augmented Reality ihre Produkte präsentieren und wo Augmented Reality in der Produktpräsentationen einen Unterschied machen kann. In diesem Kontext wird ebenfalls auf Erfolgsfaktoren von Online-Stores eingegangen, die die Kaufmotivationen von Konsumentinnen und Konsumenten positiv beeinflussen. Daran anschließend werden alle relevanten Theorien für die Studie vorgestellt, sodass die Leser:innen 
Einblicke in die Risikotheorie, das Confirmation/Disconfirmation Paradigma, die Theorie der kognitiven Dissonanz und das Model of Flow bekommen.

Die Darstellungen aus dem zweiten Kapitel werden im dritten Kapitel genutzt, um Hypothesen für die Studie abzuleiten. Es werden nacheinander die relevanten Theorien, Konzepte und Modelle aufgezeigt und in Verbindung mit der Produktpräsentation durch Augmented Reality gebracht. Beruhend darauf werden 17 Hypothesen aufgestellt, die Annahmen über den Einsatz von Augmented Reality widerspiegeln und im Folgenden getestet werden. Die Leser:innen bekommen hier insbesondere durch verschiedene Abbildungen einen guten Überblick über das Forschungsmodell, die zeigen, wie die verschiedenen Hypothesen zusammenhängen und welche statistischen Analysen für die jeweiligen Hypothesen geplant sind.

Das vierte Kapitel legt nicht nur das Forschungsdesign detailliert dar, es beschreibt außerdem statistische Grundlagen, die für die geplanten Analysen benötigt werden. So bekommen die Leser:innen Einblicke in die verschiedenen Analysemethoden und es wird deutlich, warum Varianzanalysen und Regressionsanalysen für diese Studie ausgewählt wurden. Nach der Darlegung der statistischen Grundlagen wird das Untersuchungsdesign genauer vorgestellt. Hier werden Abbildungen präsentiert, die sowohl den Einsatz von Augmented Reality zur Produktpräsentation zeigen, als auch die traditionelle Form der Präsentation von Produkten. Des Weiteren werden Informationen zur Durchführung der Studie gegeben, wie etwa der Erhebungszeitraum und die Anzahl von Probandinnen und Probanden, zudem deskriptive Statistiken. Daraufhin werden alle genutzten Fragebögen, die für die Erfassung der vorgestellten Konstrukte zum Einsatz kamen, vorgestellt und Werte wie Item-to-Item und Cronbach's Alpha präsentiert. Es wird geprüft, ob alle Voraussetzungen für die statistischen Analysen erfüllt sind und danach für jede Hypothese die relevanten statistischen Werte vorgestellt. Besonders hilfreich ist hier die Zusammenfassung durch Tab. 18, die die untersuchten Hypothesen aufzeigt und die Ergebnisse bezüglich Annahme und Verwerfung für jede Hypothese darlegt. Beruhend auf den Ergebnissen werden anschließend die Befunde diskutiert, interpretiert und in Zusammenhang mit der Literatur gesetzt. Daraus werden Implikationen für das Marketing abgeleitet und dabei das Potenzial und die Herausforderungen von Augmented Reality im E-Commerce dargestellt. Der Hauptteil des Buches endet mit einer Schlussbetrachtung, die alle relevanten Informationen und die gewonnenen Erkenntnisse zusammenfasst.

Zum Abschluss werden die verwendeten Referenzen im Literaturverzeichnis gesammelt und aufgezeigt. Der Anhang bietet schließlich eine Übersicht über die Hypothesen, stellt den kompletten verwendeten Fragebogen vor und liefert weitere Einblicke in die statistischen Auswertungen in Form von detaillierten Tabellen.

Als Fazit lässt sich zusammenfassen, dass das Buch ein sehr relevantes Thema beleuchtet, welches besonders in der heutigen Zeit sehr wichtig geworden ist. Sowohl E-Commerce als auch Augmented Reality gewinnen immer mehr an Bedeutung und lassen sich hervorragend verbinden, um das Einkaufserlebnis beim OnlineShopping zu verbessern. Den Autor:innen ist es gelungen, in diesem Bereich neue und tiefe Einblicke zu gewähren und die darunter liegenden Konzepte und Theorien zu erläutern. Die Studie wurde auf hohem Niveau durchführt, wodurch die Ergebnisse wichtige empirische Erkenntnisse für den Einsatz von Augmented Reality im E-Commerce liefern. Das Buch bringt somit einen großen Mehrwert für Ver- 
walter:innen von Online-Stores, Marketingabteilungen und an neuen Technologien interessierten Leser:innen.

Funding Open Access funding enabled and organized by Projekt DEAL.

Open Access Dieser Artikel wird unter der Creative Commons Namensnennung 4.0 International Lizenz veröffentlicht, welche die Nutzung, Vervielfältigung, Bearbeitung, Verbreitung und Wiedergabe in jeglichem Medium und Format erlaubt, sofern Sie den/die ursprünglichen Autor(en) und die Quelle ordnungsgemäß nennen, einen Link zur Creative Commons Lizenz beifügen und angeben, ob Änderungen vorgenommen wurden.

Die in diesem Artikel enthaltenen Bilder und sonstiges Drittmaterial unterliegen ebenfalls der genannten Creative Commons Lizenz, sofern sich aus der Abbildungslegende nichts anderes ergibt. Sofern das betreffende Material nicht unter der genannten Creative Commons Lizenz steht und die betreffende Handlung nicht nach gesetzlichen Vorschriften erlaubt ist, ist für die oben aufgeführten Weiterverwendungen des Materials die Einwilligung des jeweiligen Rechteinhabers einzuholen.

Weitere Details zur Lizenz entnehmen Sie bitte der Lizenzinformation auf http://creativecommons.org/ licenses/by/4.0/deed.de. 